REVIEW

\title{
Analgesia for patients with advanced disease: 2
}

\section{E J Hall, N P Sykes}

Postgrad Med J 2004;80:190-195. doi: 10.1136/pgmj.2003.015529

The first article in this series explored epidemiology and patterns of pain in advanced disease, nonpharmacological treatments, and the use of opioids to manage pain. This second article examines the use of nonopioid drugs and anaesthetic interventions for pain relief in advanced disease. It also discusses an approach to managing analgesia in dying patients and finally looks at future developments.

See end of article for authors' affiliations

......., a.,

Correspondence to: Dr Emma J Hall, St Christopher's Hospice, 51-59 Lawrie Park Road, Sydenham, London SE26 6DZ, UK; emmahall@ doctors.org.uk

Submitted

29 September 2003

Accepted

29 September 2003

\section{NON-STEROIDAL ANTI-INFLAMMATORY DRUGS AND PARACETAMOL \\ Pharmacology}

The therapeutic action of non-steroidal antiinflammatory drugs (NSAIDs) occurs mainly by inhibition of the cyclo-oxygenase-2 (COX-2) enzyme, whereas inhibition of the cyclo-oxygenase-1 (COX-1) enzyme is thought to mediate most side effects, such as gastric mucosal damage and platelet dysfunction. However, COX-2 inhibition is thought to be associated with a higher incidence of nephrotoxicity. COX inhibition reduces prostaglandin synthesis and thereby inflammation, although this mechanism only partly explains the analgesic effects of NSAIDs. The analgesic properties of paracetamol are thought to be mediated by central nervous system COX inhibition but are not completely understood.

A meta-analysis of conventional NSAIDs found no evidence for the superiority of one NSAID over another, nor indeed any data showing superiority of non-aspirin NSAIDs over aspirin or paracetamol. ${ }^{1}$ However, there is evidence that side effects differ among the NSAIDs.

The advent of COX-2 specific NSAIDs-for example, rofecoxib and celecoxib-initially promised a reduced incidence of side effects. Their use in cancer pain was extrapolated from studies in patients with osteoarthritis and rheumatoid arthritis. $^{23}$ These studies suggested that these drugs led to fewer serious complications, such as gastrointestinal perforation and bleeding. However, no studies compared the use of COX2 inhibitors with conventional NSAIDs in combination with proton pump inhibitors, and recently grave doubts have been cast on the biased reporting of results and conclusions from the CLASS study examining celecoxib. ${ }^{4}$

\section{Uses}

NSAIDs and paracetamol are used at all three steps of the analgesic "ladder". There is no evidence to suggest that NSAIDs are more effective in one type of pain than another, although they are widely used specifically for metastatic bone pain. ${ }^{1}$ Other uses include the treatment of bladder spasms, rectal pain, liver capsule pain, skin and soft tissue pain, and headache due to raised intracranial pressure.

There are few trials comparing NSAIDs with strong opioids in the treatment of cancer pain, and most of the comparative data comes from studies in fracture patients. A single dose study of ketorolac versus morphine in acute fracture pain found that ketorolac was more effective than morphine and had fewer side effects, ${ }^{5}$ but it is unlikely that this result can be extrapolated to chronic pain with repeated dosing.

\section{Commonly prescribed NSAIDs and routes of administration}

The most commonly used "traditional" NSAIDs are ibuprofen (400-800 mg three times daily) and diclofenac (up to $150 \mathrm{mg}$ daily orally or by an unlicensed route of continuous subcutaneous infusion; $100 \mathrm{mg}$ every 18 hours rectally). Ketorolac is used to manage severe cancer pain, particularly as a continuous subcutaneous infusion (unlicensed use) of 30-90 mg per 24 hours. ${ }^{6}$ It should be used for only a few days, as there is a high incidence of toxicity.

A useful alternative route if the conventional oral or rectal routes are not available is a soluble "melt", which dissolves quickly on the tonguefor example, piroxicam $20 \mathrm{mg}$ once daily.

The new COX-2 specific inhibitors rofecoxib (12.5-50 mg daily) and celecoxib (100-200 mg daily) are now in widespread use in the treatment of the pain of advanced disease, but there are concerns about an increased risk of cardiovascular events and nephrotoxicity compared with conventional NSAIDs.

COX-2 inhibitors are used preferentially over conventional NSAIDs in patients with platelet dysfunction or thrombocytopenia because specific COX-2 inhibition has little or no effect on platelet function and bleeding time. ${ }^{7}$

Topical NSAIDs can be used on small areas of soft tissue tenderness. There is evidence for their superiority over placebo creams in non-malignant pain, ${ }^{8}$ but no large trials have compared NSAIDs given by the topical route versus the oral route.

\footnotetext{
Abbreviations: COX, cyclo-oxygenase; NMDA, $N$ methyl-D aspartate; NNH, number needed to harm; NNT, number needed to treat; NSAIDs, non-steroidal anti-
} inflammatory drugs; WHO, World Health Organisation 


\section{Side effects}

The possible side effects of NSAIDs include gastric irritation, peptic ulceration, gastrointestinal bleeding, nausea and vomiting, acute renal failure, platelet dysfunction and thrombocytopenia (unusual with COX-2 inhibitors), abnormal liver function, bronchospasm (rare other than in asthmatic patients with true aspirin allergy), and fluid retention.

In patients with advanced disease it is common practice to co-prescribe proton pump inhibitors or histamine- 2 receptor antagonists with conventional NSAIDs in an attempt to reduce gastrointestinal side effects.

\section{ANTIDEPRESSANTS, ANTICONVULSANTS, AND ANTIARRHYTHMICS \\ Mechanisms}

Antidepressants are thought to enhance descending inhibitory pathways from the brain to the spinal cord, while anticonvulsants probably act on ion channels at the level of the spinal cord. ${ }^{9}$ Blockade of excitatory calcium channels is the proposed mechanism of action of gabapentin, which is one of the more commonly used anticonvulsants. Antiarrhythmics block sodium channels non-selectively in the heart and the peripheral and central nervous systems.'

There is logic in combining agents from different classes, particularly anticonvulsants and antidepressants, since they have differing mechanisms of action.

\section{Uses}

These drugs are used for the management of neuropathic pain. The World Health Organisation (WHO) guidelines suggest that they can be introduced at any step of the ladder, depending on the severity of the pain. There is no evidence to support the use of anticonvulsants for "shooting" pains and antidepressants for "burning" pains. In practice the choice of drug is often guided by side effect profiles and compliance issues-for example, antidepressants can be given once daily, whereas anticonvulsants are often given thrice daily.

Patients with neuropathic pain often need opioids and non-opioids, since there is a variable interindividual response to opioids.

Two systematic reviews have examined in detail the evidence for the effectiveness of antidepressants and anticonvulsants in neuropathic pain. ${ }^{10}{ }^{11}$ The strongest evidence for the use of these drugs is in patients with diabetic neuropathy and postherpetic neuralgia.

The number of patients needing to take the drug for one of them to have an improvement in pain relief of greater than $50 \%$ (number needed to treat, NNT) is about two for tricyclic antidepressants, whereas for serotonin specific reuptake inhibitors the NNT approaches five. The NNT for anticonvulsants is about three.

\section{Anticonvulsants}

Gabapentin (starting dose of $100 \mathrm{mg}$ thrice daily or $300 \mathrm{mg}$ nightly, increasing by $300 \mathrm{mg}$ per day every $1-2$ days to a maximum total daily dose of 2.4-3.6 g) is the only agent in this group to have a licence for malignant neuropathic pain, but its superiority in terms of efficacy and side effects remains controversial, and it is very expensive compared with other anticonvulsants. In fact, the Cochrane review found that the number needed to harm $(\mathrm{NNH}$; the number of patients who need to take the drug for one of them to experience side effects) for minor side effects was smaller for gabapentin than for carbamazepine. ${ }^{11}$ A recent phase II dose finding trial examined the use of sodium valproate in malignant disease $\mathrm{e}^{12}$ and found that, while it appeared to have some effect in malignant neuropathic pain, the incidence of side effects, such as sedation, tremor, and nausea, was high. The authors also felt that there was a large placebo effect, a factor that has been noted in previous work with patients suffering from neuropathic pain.

Gabapentin should be used with caution in patients with renal failure; the dose and dose interval should be adjustedfor example, a creatinine clearance of less than $30 \mathrm{ml}$ per min will necessitate a dose reduction of up to $80 \%$, sometimes with the drug being administered as a single daily dose every second day. ${ }^{13}$ Gabapentin has few drug interactions, which is a potential advantage. Other anticonvulsants should be used with caution in patients with hepatic impairment, and drug interactions can be problematic.

Clonazepam is a benzodiazepine with anticonvulsant properties. It has been used anecdotally to treat malignant and non-malignant neuropathic pain in advanced disease (0.25-2 mg at night). The parenteral preparation can be used subcutaneously in continuous infusion (unlicensed route and indication).

\section{Antidepressants}

The most commonly used antidepressant is amitryptiline, starting at $10-25 \mathrm{mg}$ at night and increasing up to $150 \mathrm{mg}$ if tolerated. Alternatives are doxepin and lofepramine. Patients with HIV, who may have painful neuropathy secondary to treatment as well as due to disease, appear able to tolerate much higher doses of antidepressants (probably because they tend to be younger). Some antidepressants have been effective, with reduced systemic side effects, when used topically—for example, doxepin cream. ${ }^{14}$

\section{Antiarrhythmics}

Antiarrhythmic drugs have been used to manage neuropathic pain. They are used less commonly than anticonvulsants or antidepressants in this patient group because of concerns about coexisting cardiac disease and the potential requirement for cardiac monitoring. They are probably more effective in pain caused by peripheral nerve damage, and, although evidence exists to support their use in nonmalignant pain, there is little evidence to date to support their use in cancer pain. ${ }^{15}$ Antiarrhythmics include flecainide 50-100 mg daily, mexiletine 100-200 mg daily, and lidocaine. Lidocaine (formerly lignocaine) has been given as an intravenous or subcutaneous infusion, and more recently a transdermal patch applied to the painful area has shown some promise in the treatment of postherpetic neuralgia. ${ }^{16}$

\section{N-METHYL-D-ASPARTATE RECEPTOR-CHANNEL COMPLEX ANTAGONISTS}

The dissociative anaesthetic ketamine has been used (at subanaesthetic doses) intravenously, subcutaneously, and orally in patients with intractable pain. It blocks the $N$ methyl-D-aspartate (NMDA) channel unselectively, and therefore the incidence of psychomimetic side effects is high. The incidence of central side effects can potentially be reduced by concomitant administration of an antipsychotic, such as haloperidol.

A recent Cochrane review of the use of ketamine in cancer pain found that only two randomised controlled trials fulfilled the criteria of the review and concluded that these studies were of insufficient power to provide a sufficient evidence base to support the use of ketamine in cancer pain, and further studies are required. ${ }^{17}$ Ketamine has also been used to relieve pain from critical limb ischaemia, and results from a recently published trial on this subject are encouraging. ${ }^{18}$

Amantadine, which is normally used as an antiParkinsonian therapy, is another NMDA receptor antagonist. It has shown promise in a randomised placebo controlled 
Box 1: Key points in the use of antidepressants, anticonvulsants, and antiarrhythmics

- Antidepressants. The tricyclics are more effective. Those most commonly used are amitryptiline, lofepramine, dothiepin, and doxepin. There are many side effects, including sedation, dry mouth, postural hypotension, seizures, arrhythmias, glaucoma, urine retention, and constipation.

- Anticonvulsants. Phenytoin and carbamazepine are sedating and may result in blood dyscrasias and problematic drug interactions. The most commonly used anticonvulsants are sodium valproate and gabapentin. Side effects include drowsiness (often transient), confusion, dizziness, tremor, ataxia, and hepatic dysfunction with valproate. There is little evidence to support the use of clonazepam, but it has been used, especially as a parenteral substitute when the patient is unable to swallow.

- Antiarrhythmics. The most commonly used are flecainide, mexilitene, and lidocaine. The main side effects are arrhythmias, convulsions, and hypotension.

trial in patients suffering from neuropathic pain following cancer surgery. ${ }^{19}$

Drugs such as memantine that block more specific parts of the NMDA receptor-channel complex (possibly reducing the likelihood of side effects) are undergoing trials.

\section{CORTICOSTEROIDS}

\section{Uses}

Corticosteroids are used for their anti-inflammatory and antitumour effects, particularly in breast and prostate cancers and lymphomas. They may also be used to relieve nerve compression pain (by reducing peritumoural oedema), headaches associated with raised intracranial pressure (by reducing peritumoural oedema), liver capsule pain, and bone pain.

\section{Dose and duration}

The optimal drug, dose, and duration are unclear from the evidence, but in practice dexamethasone ( $8-16 \mathrm{mg}$ in a single or divided daily dose) is often used. If there is no effect after four to seven days then the steroid should be discontinued. If there is an effect then the usual practice is to reduce the dose by 2-4 mg every three to five days, with the aim of stopping the drug altogether. Some patients cannot stop taking steroids because their symptoms recur, and, in this situation, the lowest possible maintenance dose should be the aim.

In paediatric oncology units, it is common practice to use steroid "pulses" of three to five days (strictly time limited courses, which are repeated every few weeks if the first course is effective). The aim of this approach is to reduce the incidence of side effects, but there is only anecdotal evidence to support this practice, and it is only occasionally used in adults.

\section{Side effects}

The side effects of corticosteroids include indigestion, gastrointestinal perforation (especially with the concomitant use of NSAIDs), diabetes, agitation (and, rarely, psychosis), insomnia (can be minimised by giving the drug early in the day), proximal myopathy, skin thinning, fluid retention, hypertension, fat redistribution, and osteoporosis (after a few months).

\section{ANTISPASMODIC DRUGS}

\section{Skeletal muscle relaxants}

Baclofen, tizanidine, and dantrolene have been used to relieve spasticity and painful spasms in spinal cord compression as well as in multiple sclerosis and motor neurone disease. Baclofen is used orally and intrathecally. Benzodiazepines, such as diazepam and clonazepam, have also been used for this purpose, as well as to reduce muscle tension caused by anxiety.

Injections of botulinum toxin have been used to relieve painful spasticity in patients with multiple sclerosis and may have applications in other advanced disease states, but they are not widely used in palliative care at present.

\section{Smooth muscle relaxants}

Drugs for relaxing smooth muscle include anticholinergic drugs, such as hyoscine butylbromide and hydrobromide, calcium channel blockers, such as nifedipine, and $\beta$ adrenergic agonists, such as salbutamol. Glyceryl trinitrate has also been used in this context. These drugs have been anecdotally successful in the management of intestinal colic, rectal pain, and oesophageal spasm.

\section{BISPHOSPHONATES}

Bisphosphonates inhibit osteoclastic activity, which is abnormal in metastatic bone disease. They are commonly used in the management of hypercalcaemia associated with malignancy, but also have an increasingly important role in the management of other skeletal complications associated with bone metastases. Bisphosphonates are also used in the management of osteoporosis and Paget's disease of bone, but these conditions will not be discussed further.

There is now a large body of evidence supporting the use of bisphosphonates in the treatment of bone pain caused by multiple myeloma or breast cancer. ${ }^{20}$ A recent Cochrane systematic review concluded that bisphosphonates are probably of overall benefit in metastatic bone pain, with a NNT of 11 at four weeks and seven at 12 weeks. ${ }^{21}$ However, the authors concluded that further evidence is required to demonstrate specific benefits according to the site of the primary tumour, the dose, and the type of bisphosphonate.

The optimal dose and duration of treatment remain topics for debate; the authors of another recent systematic review recommend continuing bisphosphonate treatment for as long as is clinically appropriate because of the reduction in the skeletal morbidity of metastatic bone disease brought about by these drugs. ${ }^{22}$

Commonly used bisphosphonates are clodronate (oral and intravenous routes, but the tablets have poor oral bioavailability), pamidronate (monthly intravenous infusions, each lasting one hour), and the newer more potent drug zoledronate (monthly intravenous infusions, each lasting 15 minutes).

Patients should be informed that any effect on bone pain is unlikely to occur immediately after the first bisphosphonate treatment and that they may in fact experience a pain "flare" in the first few days after treatment together with fever and arthralgia.

\section{RADIOPHARMACEUTICALS}

Strontium-89 is a radioisotope taken up by bone. It has been used successfully in the management of refractory bone pain in prostate cancer. ${ }^{23}$ It is extremely expensive and can cause severe myelosuppression. Rhenium-186 hydroxyethylidene diphosphonate is another radioisotope currently under investigation in patients with prostate cancer. Radioisotopes may also be helpful in the management of bone metastases from carcinoid tumours. 


\section{OTHER ADJUVANT DRUGS}

Topical capsaicin cream is derived from hot chilli peppers and when applied over a period of time depletes substance P from unmyelinated $C$ fibres. It is licensed for the treatment of osteoarthritis, postherpetic neuralgia, and diabetic neuropathy. It has shown efficacy in the treatment of post-surgical neuropathic pain in patients with cancer, ${ }^{24}$ but side effects included high levels of burning, redness, and cough. The length of time for onset of action (up to two weeks) makes it impractical for patients with short prognoses.

Clonidine is an $\alpha$-adrenergic agonist and is normally used in spinal infusions to enhance analgesia, but it has been used anecdotally to treat neuropathic pain with a sympathetic component. Its use is limited by its propensity to cause profound hypotension.

Entonox, a medical gas containing 50\% oxygen and 50\% nitrous oxide, is occasionally used to relieve short lived pain, particularly that associated with painful dressing changes.

\section{INVASIVE ANAESTHETIC TECHNIQUES}

\section{Spinal opioids and local anaesthetics}

Delivering opioids directly into the epidural space or cerebrospinal fluid allows much smaller doses to be used than when they are administered orally, with a reduction in adverse effects. In practice, opioids are often combined with local anaesthetics (bupivacaine or the newer agent ropivacaine) and sometimes clonidine, an $\alpha$-adrenergic agonist that can enhance analgesia. Although case series report high success rates with spinal analgesia, it has been difficult to demonstrate superiority over conventional parenteral routes in comparative studies. ${ }^{25}$

\section{Uses}

Spinal analgesia has been used in the management of neuropathic pain from spinal cord or nerve root compression, incident pain from pathological fractures in patients unfit for surgery, and peripheral arterial limb ischaemia.

Although continuous spinal infusions have been extensively used in the inpatient setting, managing them in the patient's home is fraught with difficulties, particularly in finding appropriately trained staff to manage the pumps. However, some areas do have well established protocols and teams with expertise in this subject. There is wide variation in clinical practice, but the recent publication of guidelines on managing spinal analgesia may provide helpful clarification. ${ }^{26}$

\section{Side effects}

Side effects of spinal analgesia include hypotension, respiratory depression, urine retention, pruritus, meningitis, and epidural abscesses.

\section{Regional analgesia}

Regional analgesia involves injecting local anaesthetic and a steroid into painful areas-for example, trigger points and intercostal and rib blocks. Interpleural catheters delivering bupivacaine have been used to manage painful pleural cancers and invasion of the chest wall, and a small case series reports this as a useful technique. ${ }^{27}$ Brachial plexus and suprascapular blocks have been used in the treatment of upper limb pain, while lumbar plexus blocks can be helpful in managing pain arising from the hip.

\section{Neurolytic blocks}

In neurolytic blocks, neural pathways are irreversibly damaged with the intention of disrupting pain transmission. Techniques include using phenol, heat, cold, and surgical lesioning. All of these techniques must be performed by experienced operators, which restricts their use. Examples include coeliac plexus block with phenol for upper abdominal visceral pain, cervical cordotomy for unilateral pain-for example, in mesothelioma, ${ }^{28}$ and lumbar sympathectomy with phenol for ischaemic limb pain or rectal pain.

\section{THE FINAL FEW DAYS AND HOURS}

This article has so far dealt with analgesia in patients with terminal disease who may still have several months to live. In the very last stages of a person's life, emphasis should be placed on achieving optimal pain control while keeping medication as simple as possible.

The oral route may become unavailable, and drugs may need to be delivered subcutaneously using a syringe driver. The dose of opioid required for subcutaneous delivery will need to be calculated-for example, converting from oral morphine to subcutaneous diamorphine requires that the 24 hour morphine dose is divided by three. Suitable rescue doses of opioid as well as antiemetics and anxiolytics should be prescribed.

If the patient has neuropathic pain then it may be acceptable simply to increase the opioid dose. Clonazepam is sometimes used as a parenteral alternative to neuropathic agents but there is little evidence for its efficacy.

An antiemetic may be required, as may an anxiolytic if the patient is frightened.

It is essential to communicate medication changes to the patient and their family.

\section{FUTURE DIRECTIONS}

Future directions can be summed up as:

- More selective NMDA antagonists.

- A small uncontrolled study of 12 patients with cancer pain highlighted the potential of intravenous magnesium sulphate as a therapeutic option in the management of neuropathic pain. ${ }^{30}$ Magnesium ions block the NMDA channel in the resting state. However, it may be helpful only in patients with low plasma concentrations of magnesium.

- More selective sodium channel blockers (to avoid the side effects of local anaesthetics).

\section{Box 2: Summary of pharmacological}

interventions for pain

1. Disease modifying: palliative chemotherapy ${ }^{29}$ and radiopharmaceuticals; steroids and bisphosphonates can also be disease modifying.

2. NSAIDs and paracetamol: used for bone pain, bladder and rectal spasms, liver capsule pain, and headache.

3. Adjuvants for neuropathic pain: antidepressants, anticonvulsants, antiarrhythmics (including lidocaine), corticosteroids, capsaicin cream, clonidine, and NMDA antagonists (for example, ketamine and amantadine).

4. Opioids ${ }^{29}$ : used for all types of pain; administered by various routes including spinal.

5. Benzodiazepines, baclofen, dantrolene, botulinum toxin: used for skeletal muscle spasms, painful spasticity, and muscle tension induced by anxiety.

6. Anticholinergics, calcium channel antagonists, $\beta$-adrenergic agonists: used to treat smooth muscle spasms in intestinal colic, rectal pain, and oesophageal spasm.

7. Interventional anaesthetic techniques: used for neuropathic pain, fractures, severe visceral pain, and critical limb ischaemia.

8. Entonox: used for short lived pain (for example, pain caused by dressing changes). 


\begin{tabular}{|l|l|}
\cline { 3 - 3 } \multicolumn{1}{c|}{} & $\begin{array}{l}\text { Step 3 } \\
\text { "Strong opioid" for } \\
\text { severe pain +/-non- } \\
\text { opioid +/-adjuvant }\end{array}$ \\
\cline { 2 - 2 } \multicolumn{1}{l|}{$\begin{array}{l}\text { Step 2 } \\
\text { "Mild opioid" for } \\
\text { mild-moderate pain } \\
\text { +/-non-opioid +/- } \\
\text { adjuvant }\end{array}$} & \\
$\begin{array}{l}\text { Non-opioid } \\
+/- \text { adjuvant }\end{array}$ & $\begin{array}{l}\text { General/neurosurgery/orthopaedic surgery } \\
\text { Interventional anaesthetic techniques } \\
\text { TENS/acupuncture/complementary therapy }\end{array}$ \\
\hline $\begin{array}{l}\text { Disease-modifying treatment } \\
\text { Chemotherapy/radiotherapy/radiopharmaceuticals/steroids/ } \\
\text { bisphosphonates }\end{array}$ \\
\hline $\begin{array}{l}\text { Address psychological, emotional, spiritual, social, financial } \\
\text { distress }\end{array}$ \\
\hline
\end{tabular}

Figure 1 Modified analgesic ladder (reproduced with permission from The palliative care handbook. 5th Ed. Forest Holme and Wessex Specialist Palliative Care units. Dorset Cancer Network).

- Cannabinoids for neuropathic pain (although a recent systematic review showed no benefit of cannabinoids over codeine in the management of non-neuropathic pain $\left.^{31}\right)$.

- In chronic opioid administration levels of the neuropeptide cholecystokinin are raised, and this may be responsible for opioid tolerance and non-responsiveness. Cholecystokinin receptor antagonists might be able to combat this.

- Levels of the nucleoside adenosine are reduced in chronic pain states, and adenosine analogues are the subject of trials.

- Single molecules with NMDA antagonism combined with opioid agonism.

- Novel agonists for the ORL-1 opioid receptor.

- Nitric oxide donating NSAIDs and dual cyclo-oxygenaselipo-oxygenase inhibitors. ${ }^{32}$

\section{Box 3: Key references for parts 1 and 2}

- Scottish Intercollegiate Guidelines Network. Control of pain in patients with cancer. No 44. Edinburgh: Scottish Intercollegiate Guidelines Network (SIGN), 2000 (www.sign.ac.uk/guidelines).

- McQuay HJ, Tramer MR, Nye BA, et al. A systematic review of antidepressants in neuropathic pain. Pain 1996;68:217-27.

- Wiffen P, Collins S, McQuay H, et al. Anticonvulsant drugs for acute and chronic pain. Cochrane Library. Issue 2. Oxford: Update Software, 2002.

- Hanks GW, de Conno F, Cherny N, et al. Expert Working Group of the Research Network of the European Association for Palliative Care. Morphine and alternative opioids in cancer pain: the EAPC recommendations. Br J Cancer 2001 ;84:587-93.

- Cherny N, Ripamonti C, Pereira J, et al. Expert working group of the European Association for Palliative Care. Strategies to manage the adverse effects of oral morphine: an evidence-based report. J Clin Oncol $2001 ; 19: 2542-54$.

\section{Box 4: Questions (answers at end of references)}

1. Which analgesics are used at step 1 of the WHO analgesic ladder?

2. Which strong opioid should never be used in the management of cancer pain?

3. List the drugs that are used as adjuvant analgesics.

4. Give an example of a non-pharmacological technique for managing pain.

5. When prescribing morphine for someone with renal failure, what adjustments should you make?

6. A patient taking $240 \mathrm{mg}$ per day of oral morphine is dying and requires a subcutaneous infusion. How much diamorphine per day should they receive?

\section{CONCLUSION}

This review sets out in detail the therapeutic options available for analgesia in patients with advanced disease. The key to managing pain lies in assessing the patient and taking into account both psychosocial and physical aspects. It should be remembered that more than one pain may be present. The WHO guidelines using the analgesic ladder should form a guide for management, but disease modifying treatments and surgical and anaesthetic techniques should also be remembered, as should factors that may increase the total suffering caused by pain. Figure 1 summarises the overall approach to pain management.

\section{Authors' affiliations \\ E J Hall, N P Sykes, St Christopher's Hospice, London, UK}

\section{REFERENCES}

1 Eisenberg E, Berkey CS, Carr DB, et al. Efficacy and safety of nonsteroidal antiinflammatory drugs for cancer pain: a meta-analysis. J Clin Oncol 1994; 12:2756-65.

2 Silverstein FE, Faich G, Goldstein JL, et al. Gastrointestinal toxicity with celecoxib vs. nonsteroidal antiinflammatory drugs for osteoarthritis and rheumatoid arthritis: the CLASS study: a randomised control trial. Celecoxib Long-term Arthritis Safety Study. JAMA 2000;284:1247-55.

3 Bombardier C, Laine L, Reicin A, et al. Comparison of upper gastrointestinal toxicity of rofecoxib and naproxen in patients with rheumatoid arthritis. VIGOR study group. N Engl J Med 2000;343:1520-8.

4 Juni P, Rutjes AWS, Dieppe PA. Are selective COX 2 inhibitors superior to traditional non-steroidal anti-inflammatory drugs? BMJ 2002;324:1287-8.

5 Rainer TH, Jacobs P, Ng YC, et al. Cost-effectiveness of intravenous ketorolac and morphine for treating pain after limb injury: double-blind, randomised, controlled trial. BMJ 2000;321:374-87.

6 Middleton RK, Lyle JA, Berger DL. Ketorolac continuous infusion: a case report and review of the literature. J Pain Symptom Manage 1996; 12:190-4.

7 Leese PT, Hubbard RC, Karim A, et al. Effects of celecoxib, a novel cyclooxygenase- 2 inhibitor, on platelet function in healthy adults: a randomised, controlled trial. J Clin Pharmacol 2000;40:124-32.

8 Moore RA, Tramer MR, Carroll D, et al. Quantitative systematic review of topically applied non-steroidal anti-inflammatory drugs. BMJ 1998;316:333-8.

9 Jensen TS, Wilson PR, Rice ASC, eds. Chronic pain. London: Arnold, 2003:241, 251, 255.

10 McQuay HJ, Tramer M, Nye BA, et al. A systematic review of antidepressants in neuropathic pain. Pain 1996;68:217-27.

11 Wiffen P, Collins S, McQuay H, et al. Anticonvulsant drugs for acute and chronic pain. Cochrane Library. Issue 2. Oxford: Update Software, 2002.

12 Hardy JR, Rees EAJ, Gwilliam B, et al. A phase II study to establish the efficacy and toxicity of sodium valproate in patients with cancer-related neuropathic pain. J Pain Symptom Manage $2001 ; 21: 204-9$.

13 Pfizer. Available at: www.pfizer.com/download/uspi_neurontin.pdf.

14 McCleane G. Topical application of doxepin hydrochloride, capsaicin and a combination of both produces analgesia in chronic human neuropathic pain: a randomised, double-blind, placebo-controlled study. Br J Clin Pharmacol 2000;49:574-9.

15 Kalso E, Tramer MR, McQuay HJ, et al. Systemic local-anaesthetic type drugs in chronic pain: a systematic review. Eur J Pain 1998;2:3-14. 
16 Galer BS, Rowbotham MC, Perander J, et al. Topical lidocaine patch relieves postherpetic neuralgia more effectively than a vehicle topical patch: results of an enriched enrolment study. Pain 1999;80:533-8.

17 Bell R, Eccleston C, Kalso E. Ketamine as an adjuvant for cancer pain. Cochrane Database Syst Rev 2003;(1):CD003351.

18 Mitchell AC, Fallon MT. A single infusion of intravenous ketamine improves pain relief in patients with critical limb ischaemia: results of double blind randomised controlled trial. Pain 2002;97:275-81.

19 Pud D, Eisenberg E, Spitzer A, et al. The NMDA receptor antagonist amantadine reduces surgical neuropathic pain in cancer patients: a doubleblind, randomised, placebo-controlled trial. Pain 1998;75:349-54.

20 Fulfaro F, Casuccio A, Ticozzi C, et al. The role of bisphosphonates in the treatment of painful metastatic bone disease: a review of phase III trials. Pain 1998:78:157-69.

21 Wong R, Wiffen PJ. Bisphosphonates for the relief of pain secondary to bone metastases. Cochrane Database Syst Rev 2002;(2):CD002068

22 Ross JR, Saunders Y, Edmonds PM, et al. Systematic review of the role of bisphosphonates in skeletal morbidity in metastatic cancer. BMJ 2003:327:469-72.

23 Quilty PM, Kirk D, Bolger JJ, et al. A comparison of the palliative effects of strontium-89 and external beam radiotherapy in metastatic prostate cancer Radiother Oncol 1994;31:33-40.

24 Ellison N, Loprinzi CL, Kugler J, et al. A phase III placebo-controlled trial of capsaicin cream in the management of surgical neuropathic pain in cancer patients. J Clin Oncol 1997; 15:2974-80.

25 Williams J. Critical appraisal of invasive therapies used to treat chronic pain and cancer pain. Eur J Palliat Care 2000:7:121-5.

26 Bennett G, Burchiel K, Buchser E, et al. Clinical guidelines for intraspinal infusions: report of an expert panel. Polyanalgesic Consensus Conference 2000. J Pain Symptom Manage 2000;20:537-43.

27 Amesbury B, O'Riordan J, Dolin S. The use of interpleural analgesia using bupivacaine for pain relief in advanced cancer. Palliat Med 1999;13:153-8.
28 Jackson $M B$, Pounder D, Price C, et al. Percutaneous cervical cordotomy for the control of pain in patients with pleural mesothelioma. Thorax 1999;54:238-41

29 Hall EJ, Sykes NP. Analgesia for patients with advanced disease: 1. Postgrad Med J 2004:80:148-54.

30 Crosby V, Wilcock A, Corcoran R. The safety and efficacy of a single dose $(500 \mathrm{mg}$ or $1 \mathrm{~g}$ ) of intravenous magnesium sulfate in neuropathic pain poorly responsive to strong opioid analgesics in patients with cancer. J Pain Symptom Manage 2000; 19:35-9.

31 Campbell FA, Tramer MR, Carroll D, et al. Are cannabinoids an effective and safe treatment option in the management of pain? A qualitative systematic review. BMJ 2001;323:13-16.

32 Skelly MM, Hawkey CJ. Potential alternatives to COX 2 inhibitors. BMJ 2002;324:1289-90.

\section{ANSWERS}

1. Non-steroidals, paracetamol, and adjuvant drugs.

2. Pethidine: it is too short acting, and toxic metabolites accumulate with repeated dosing.

3. Antidepressants, anticonvulsants, antiarrhythmics, antispasmodics, steroids, bisphosphonates, and antibiotics.

4. Prophylactic orthopaedic surgery to manage lytic bone secondaries.

5. Increase the dose interval to 6-8 hours, and reduce the dose by $30 \%-50 \%$.

6. $80 \mathrm{mg}$ of diamorphine per day.

\section{$\mathrm{ECHO}$}

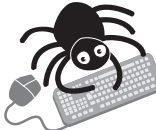

Please visit the Postgraduate Medical Journal website [www. postgradmedi com] for a link to the full text of this article.
Proposed risk stratification in upper gastrointestinal haemorrhage: Is hospitalisation essential?

A E Courtney, R M S Mitchell, L Rocke, B T Johnston

Aims: Patients with upper gastrointestinal haemorrhage (UGIH) are usually admitted to hospital regardless of the severity of the bleed. The aim of this study was to identify patients who could be safely managed without hospitalisation and immediate inpatient endoscopy. Methods: Based on a literature review, a protocol was devised using clinical and laboratory data regarded as being of prognostic value. A retrospective observational study of consecutive patients who attended the emergency department (ED) with UGIH was conducted during one calendar month.

Results: Fifty four patients were identified of whom $44(81 \%)$ were admitted. Twelve suffered an adverse event. One of the 10 patients ( $10 \%)$ initially discharged from the ED was later admitted. Strict implementation of the protocol would have resulted in safe discharge of a further 15 patients, ( $34 \%$ of those admitted), and a saving of an estimated 37 bed days per month.

Conclusions: Patients at low risk from UGIH may be identified in the ED. If validated, this protocol may improve patient management and resource utilisation.

A Emergency Medicine Journal 2004;21:39-40. 